\title{
Existence and exponential stability of almost-periodic solutions for MAM neural network with distributed delays on time scales
}

\author{
GAO Jin ${ }^{1,2} \quad$ WANG Qi-ru² $\quad$ LIN Yuan ${ }^{3, *}$
}

\begin{abstract}
This paper is concerned with multidirectional associative memory neural network with distributed delays on almost-periodic time scales. Some sufficient conditions on the existence, uniqueness and the global exponential stability of almost-periodic solutions are established. An example is presented to illustrate the feasibility and effectiveness of the obtained results.
\end{abstract}

\section{$\S 1 \quad$ Introduction}

In the past few decades, neural networks have attracted people's high degree of attention. There have been a lot of studies concerning the stability of the neural network system. One can refer to [4-6] and the references therein. The multidirectional associative memory (MAM) neural network was proposed by Hagiwara [10] in 1990. The MAM neural network is an extension of BAM neural network model. It has been found wide applications in image denoising, speech recognition, pattern recognition and intelligent information processing. In recent years, the MAM neural networks have been discussed in many papers [11][15-17]. form

In [16], the authors considered MAM neural networks with distributed delays of the following

$$
\frac{d x_{k i}}{d t}=-a_{k i}(t) x_{k i}(t)+\sum_{p=1, p \neq k}^{m} \sum_{j=1}^{n_{p}} w_{p j}^{k i}(t) f_{p j}\left(\int_{0}^{+\infty} g_{p j}^{k i}(s) x_{p j}(t-s) d s\right)+I_{k i}(t),
$$

where $t \geq 0, k=1,2, \ldots, m, i=1,2, \ldots, n_{k}, x_{k i}(t)$ denotes the membrane voltage of the $i$ th neuron in the field $k$ at time $t, a_{k i}>0$ denotes the decay rate of the $i$ th neuron in the field $k$, $f_{p j}(\cdot)$ is a neuronal activation function of the $j$ th neuron in the field $p, w_{p j}^{k i}$ is the connection

\footnotetext{
Received: 2018-03-16. Revised: 2019-12-06.

MR Subject Classification: 26E70, 39A24.

Keywords: multidirectional associative memory neural networks, time scales, almost-periodic solutions, exponential stability.

Digital Object Identifier(DOI): https://doi.org/10.1007/s11766-021-3606-z.

Supported by the National Natural Science Foundation of China $(11671406,12071491)$ and the Research Fund of Shenzhen Institute of Information Technology (QN201703).

*Corresponding author.

(C)The Author(s) 2021, corrected publication June 2021.

The original version of this article was revised due to a retrospective Open Access order.
} 
weight from the $j$ th neuron in the field $p$ to the $i$ th neuron in the field $k, I_{k i}$ is the external input of the $i$ th neuron in the field $k$.

The theory of time scales was created in order to unify the study of continuous and discrete analysis. It has a tremendous potential for applications and has recently received much attention. However, only little attention has been paid towards the existence, uniqueness and stability of almost-periodic solutions to MAM neural networks on time scales, and thus, it is important and necessary to study them. We found that, the delta derivative and the nabla derivative are different unless $\mathbb{T}=\mathbb{R}$. Especially, when $\mathbb{T}=\mathbb{Z}$, the nabla derivative $y^{\nabla}$ is the backward difference while the delta derivative $y^{\Delta}$ is the forward difference. Then in $[8,9]$, we extended the almost-periodic theory on time scales with the delta derivative to that with the nabla derivative, and then derived some sufficient conditions ensuring the existence, uniqueness and exponential stability of almost-periodic solutions for the neural networks on time scales.

Motivated by the above mentioned studies, in the present paper we shall consider MAM neural networks with distributed delays on time scales of the following form

$$
x_{k i}^{\nabla}(t)=-a_{k i}(t) x_{k i}(t)+\sum_{p=1, p \neq k}^{m} \sum_{j=1}^{n_{p}} w_{p j}^{k i}(t) f_{p j}\left(\int_{0}^{+\infty} g_{p j}^{k i}(s) x_{p j}(t-s) \nabla s\right)+I_{k i}(t),
$$

where $t \in[0,+\infty)_{\mathbb{T}}, \mathbb{T}$ is an almost-periodic time scale, $[0,+\infty)_{\mathbb{T}}=\{t \mid t \in[0,+\infty) \cap \mathbb{T}\}, k=$ $1,2, \ldots, m, i=1,2, \ldots, n_{k}$.

The initial condition of $(1.2)$ is

$$
x_{k i}(s)=\theta_{k i}(s), s \in(-\infty, 0]_{\mathbb{T}},
$$

where $\theta_{k i}(s) \in C^{1}\left((-\infty, 0]_{\mathbb{T}}, \mathbb{R}\right),(-\infty, 0]_{\mathbb{T}}=\{t \mid t \in(-\infty, 0] \cap \mathbb{T}\}, k=1,2, \ldots, m, i=1,2, \ldots, n_{k}$.

The rest of this paper is organized as follows. In Section 2, we introduce some notations and definitions and state some preliminary results. These results play an important role in Sections 3 and 4. In Section 3, we establish some sufficient conditions for the existence and uniqueness of almost-periodic solutions of (1.2). In Section 4, we prove that the almost-periodic solution obtained in Section 3 is globally exponentially stable. In Section 5, we give an example to illustrate the feasibility and effectiveness of our results obtained in previous sections. In Section 6 , we give a conclusion of this paper.

\section{$\S 2 \quad$ Preliminaries and lemmas}

In this section, we introduce some notations and definitions and state some preliminary results in $[1-3][7-9][12-14]$.

Let $\mathbb{T}$ be a nonempty closed subset (time scale) of $\mathbb{R}$. For $t \in \mathbb{T}$, we define the forward and backward jump operators $\sigma, \rho: \mathbb{T} \rightarrow \mathbb{T}$, respectively, by

$$
\sigma(t)=\inf \{s \in \mathbb{T}: s>t\}, \quad \rho(t)=\sup \{s \in \mathbb{T}: s<t\} .
$$

A point $t \in \mathbb{T}$ is called left-dense if $t>\inf \mathbb{T}$ and $\rho(t)=t$, left-scattered if $\rho(t)<t$, rightdense if $t<\sup \mathbb{T}$ and $\sigma(t)=t$, and right-scattered if $\sigma(t)>t$. If $\mathbb{T}$ has a left-scattered maximum $m$, then $\mathbb{T}^{k}:=\mathbb{T} \backslash\{m\}$; otherwise $\mathbb{T}^{k}:=\mathbb{T}$. If $\mathbb{T}$ has a right-scattered minimum $m$, then $\mathbb{T}_{k}:=\mathbb{T} \backslash\{m\}$; otherwise $\mathbb{T}_{k}:=\mathbb{T}$. The backwards graininess $\nu: \mathbb{T}_{k} \rightarrow[0, \infty)$ is defined by $\nu(t)=t-\rho(t)$.

A function $f: \mathbb{T} \rightarrow \mathbb{R}$ is called left-dense continuous or ld-continuous provided it is continuous at left-dense point in $\mathbb{T}$ and its right-side limits exist (finite) at right-dense points in 
T.

For a function $f: \mathbb{T} \rightarrow \mathbb{R}$ and $t \in \mathbb{T}_{k}$, we define the nabla derivative of $f$ at $t$, denoted $f^{\nabla}(t)$, to be the number (provided it exists) with the property that given any $\varepsilon>0$, there is a neighborhood $U$ of $t$ such that

$$
\left|f(\rho(t))-f(s)-f^{\nabla}(t)(\rho(t)-s)\right| \leq \varepsilon|\rho(t)-s| \text { for all } s \in U .
$$

If $f$ is ld-continuous, then there is a function $F$ such that $F^{\nabla}(t)=f(t)$. In this case, we define

$$
\int_{a}^{b} f(t) \nabla t=F(b)-F(a) .
$$

The function $p$ is $\nu$-regressive if $1-\nu(t) p(t) \neq 0$ for all $t \in \mathbb{T}_{k}$. Define the $\nu$-regressive class of functions on $\mathbb{T}_{k}$ to be $R_{\nu}=\{p: \mathbb{T} \rightarrow \mathbb{R} \mid p$ is ld-continuous and $\nu$-regressive $\}$.

We define the set $R_{\nu}^{+}$of all positively $\nu$-regressive elements by $R_{\nu}^{+}=R_{\nu}^{+}(\mathbb{T}, \mathbb{R})=\left\{p \in R_{\nu}\right.$ : $1-\nu(t) p(t)>0$, for all $t \in \mathbb{T}\}$.

If $p \in R_{\nu}$, then we define the nabla exponential function by

for $s, t \in \mathbb{T}$, where the $\nu$-cylinder transformation

$$
\hat{e}_{p}(t, s)=\exp \left\{\int_{s}^{t} \hat{\xi}_{\nu(\tau)}(p(\tau)) \nabla \tau\right\}
$$

$$
\hat{\xi}_{h}(z)= \begin{cases}-\frac{\log (1-h z)}{h} & \text { if } h \neq 0, \\ z & \text { if } h=0 .\end{cases}
$$

Definition 2.1 If $p, q \in R_{\nu}$, then we define a circle plus addition by

$$
\left(p \oplus_{\nu} q\right)(t):=p(t)+q(t)-p(t) q(t) \nu(t)
$$

for all $t \in \mathbb{T}_{k}$. For $p \in R_{\nu}$, define a circle minus $p$ by

$$
\ominus_{\nu} p:=-\frac{p}{1-\nu p} .
$$

Lemma 2.1( $[1,3])$ Let $p, q \in R_{\nu}$ and $s, t, r \in \mathbb{T}$. Then

(i) $\hat{e}_{0}(t, s) \equiv 1 \quad$ and $\quad \hat{e}_{p}(t, t) \equiv 1$

(ii) $\hat{e}_{p}(\rho(t), s)=(1-\nu(t) p(t)) \hat{e}_{p}(t, s)$;

(iii) $\hat{e}_{p}(t, s)=1 / \hat{e}_{p}(s, t)=\hat{e}_{\ominus \nu} p(s, t)$;

(iv) $\hat{e}_{p}(t, r) \hat{e}_{p}(r, s)=\hat{e}_{p}(t, s)$;

(v) $\left(\hat{e}_{p}(t, s)\right)^{\nabla}=p(t) \hat{e}_{p}(t, s)$.

Lemma 2.2( $[1,3])$ Let $f, g$ be nabla differentiable functions on $\mathbb{T}$. Then

(i) $\left(\beta_{1} f+\beta_{2} g\right)^{\nabla}=\beta_{1} f^{\nabla}+\beta_{2} g^{\nabla}$, for any constants $\beta_{1}, \beta_{2}$;

(ii) $(f g)^{\nabla}(t)=f^{\nabla}(t) g(t)+f(\rho(t)) g^{\nabla}(t)=f(t) g^{\nabla}(t)+f^{\nabla}(t) g(\rho(t))$;

(iii) If $f$ and $f^{\nabla}$ are continuous, then $\left(\int_{a}^{t} f(t, s) \nabla s\right)^{\nabla}=f(\rho(t), t)+\int_{a}^{t} f^{\nabla}(t, s) \nabla s$.

Lemma 2.3( $[1,3])$ Assume $p \in \mathbb{R}_{\nu}$ and $t_{0} \in \mathbb{T}$. If $1-\nu(t) p(t)>0$ for $t \in \mathbb{T}$, then $\hat{e}_{p}\left(t, t_{0}\right)>0$ for all $t \in \mathbb{T}$.

Definition 2.2 A time scale $\mathbb{T}$ is called an almost-periodic time scale if

$$
\Pi:=\{\tau \in \mathbb{R}: t \pm \tau \in \mathbb{T}, \forall t \in \mathbb{T}\} \neq\{0\} .
$$

Let $\mathbb{T}=\bigcup_{k \in \mathbb{Z}}[k(a+b), k(a+b)+b], a \neq b$,then $\mathbb{T}$ is an almost-periodic time scale. If $b=0, a=1$, then $\mathbb{T}=\mathbb{Z}$; if $b=1, a=0$, then $\mathbb{T}=\mathbb{R}$.

Definition 2.3 Let $\mathbb{T}$ be an almost-periodic time scale and $\mathbb{E}=\mathbb{R}$ or $\mathbb{C}$. A function $f \in$ $C\left(\mathbb{T}, \mathbb{E}^{n}\right)$ is called an almost-periodic function if the $\varepsilon$-translation set of $f$

$$
E\{\varepsilon, f\}=\{\tau \in \Pi:|f(t+\tau)-f(t)|<\varepsilon, \forall t \in \mathbb{T}\}
$$


is a relatively dense set in $\mathbb{T}$ for all $\varepsilon>0$; that is, for any given $\varepsilon>0$, there exists a constant $l(\varepsilon)>0$ such that each interval of length $l(\varepsilon)$ contains a $\tau(\varepsilon) \in E\{\varepsilon, f\}$ such that

$$
|f(t+\tau)-f(t)|<\varepsilon, \quad \forall t \in \mathbb{T} .
$$

$\tau$ is called the $\varepsilon$-translation number of $f$ and $l(\varepsilon)$ is called the inclusion length of $E\{\varepsilon, f\}$.

Definition 2.4 Let $A(t)$ be an $n \times n$ matrix-valued function on $\mathbb{T}$. Then the linear system

$$
x^{\nabla}(t)=A(t) x(t), \quad t \in \mathbb{T}
$$

is said to admit an exponential dichotomy on $\mathbb{T}$ if for some (and hence for any) fundamental solution matrix $X(t)$, there exist positive constants $k_{i}, \alpha_{i}, i=1,2$, and projection $P$ such that

$$
\begin{aligned}
& \left|X(t) P X^{-1}(s)\right| \leq k_{1} \hat{e}_{\ominus_{\nu} \alpha_{1}}(t, s), \quad s, t \in \mathbb{T}, t \geq s, \text { and } \\
& \left|X(t)(I-P) X^{-1}(s)\right| \leq k_{2} \hat{e}_{\ominus_{\nu} \alpha_{2}}(s, t), \quad s, t \in \mathbb{T}, t \leq s
\end{aligned}
$$

where $|\cdot|$ is a matrix norm.

Consider the following almost-periodic system

$$
x^{\nabla}(t)=A(t) x(t)+g(t), \quad t \in \mathbb{T},
$$

where $A(t)$ is an almost-periodic matrix function, $g(t)$ is an almost-periodic vector function.

Lemma 2.4( [8]) Let $X(t)$ be a fundamental solution matrix of (2.1). If (2.1) admits an exponential dichotomy, then (2.2) has a unique almost-periodic solution as follows:

$$
x(t)=\int_{-\infty}^{t} X(t) P X^{-1}(\rho(s)) g(s) \nabla s-\int_{t}^{+\infty} X(t)(I-P) X^{-1}(\rho(s)) g(s) \nabla s .
$$

Lemma 2.5( [8]) Assume $c_{i}(t)$ is almost-periodic, $c_{i}(t) \in R_{\nu}^{+}, c_{i}(t)>0$ for $i=1,2, \ldots, n, t \in \mathbb{T}$, and

Then the following linear system

$$
\min _{1 \leq i \leq n}\left\{\inf _{t \in \mathbb{T}} c_{i}(t)\right\}=\bar{m}>0
$$

$$
x^{\nabla}(t)=\operatorname{diag}\left(-c_{1}(t),-c_{2}(t), \ldots,-c_{n}(t)\right) x(t)
$$

admits an exponential dichotomy on $\mathbb{T}$.

Lemma 2.6( $[16])$ Assume that a square matrix $A=\left(a_{i j}\right)_{n \times n}$ satisfies $a_{i j}<1$ and $a_{i j} \geq$ $0(i, j=1,2, \ldots, n)$. Then the matrix $E_{n}-A$ is a nonsingular $M$ matrix if and only if the spectral radius $\widetilde{\rho}(A)<1$, where the matrix $E_{n}$ is an identity matrix.

Set the vector $x(t)=\left(x_{11}(t), \ldots, x_{1 n_{1}}(t), \ldots, x_{m 1}(t), \ldots, x_{m n_{m}}(t)\right)^{T} \triangleq \operatorname{col}\left\{x_{k i}(t)\right\}$, and $|x(t)| \triangleq \operatorname{col}\left\{\left|x_{k i}(t)\right|\right\}$. We denote $x(t) \leq y(t)$ if $x_{k i}(t) \leq y_{k i}(t)$ for $k=1,2, \ldots, m, i=$ $1,2, \ldots, n_{k}$, and $\sup _{t \in[0,+\infty)_{\mathbb{T}}} x(t) \triangleq \operatorname{col}\left\{\sup _{t \in[0,+\infty)_{\mathbb{T}}} x_{k i}(t)\right\}$. Let $N$ denote the total number of neurons in the MAM neural network $(1.2), N=\sum_{k=1}^{m} n_{k}$. For any $x(t) \in \mathbb{R}^{N}$, its norm $\|x(t)\|=$ $\max _{\substack{k=1,2, \ldots, m \\ i=1,2, \ldots, n_{k}}}\left|x_{k i}(t)\right|$. Let $S=\left\{\varphi(t)=\left(\varphi_{11}(t), \ldots, \varphi_{1 n_{1}}(t), \ldots, \varphi_{m 1}(t), \ldots, \varphi_{m n_{m}}(t)\right)^{T} \in \mathbb{R}^{N}\right.$, where $\varphi_{k i}(t)$ is an almost periodic function on $\left.[0,+\infty)_{\mathbb{T}}, k=1,2, \ldots, m, i=1,2, \ldots, n_{k}\right\}$. For any $\varphi(t) \in S$, we define $\|\varphi\|=\sup _{t \in[0,+\infty)_{\mathbb{T}}}\|\varphi(t)\|$, then $S$ is a Banach space.

Definition 2.5 Let $N$ is a neighborhood of $t$, for $t \in \mathbb{T}$. Then $D^{-} u^{\nabla}(t)$ is called Dini nabla derivative, if there exists a left neighborhood $N(\varepsilon) \subset N$ of $t$ such that

for $s \in N(\varepsilon), s<t$.

$$
\frac{u(\rho(t))-u(s)}{\rho(t)-s}<D^{-} u^{\nabla}(t)+\varepsilon,
$$


Definition 2.6 An almost-periodic solution $x^{*}(t)=\operatorname{col}\left\{x_{k i}^{*}(t)\right\}$ of (1.2) with initial value $\varphi^{*}(s)=\operatorname{col}\left\{\varphi_{k i}^{*}(s)\right\}$ is said to be globally exponentially stable, if there exist a positive constant $M>1$ and a positive constant $\lambda>0$ such that for an arbitrary solution $x(t)=\operatorname{col}\left\{x_{k i}(t)\right\}$ with initial value $\varphi(s)=\operatorname{col}\left\{\varphi_{k i}(s)\right\}$,

$$
\left\|x(t)-x^{*}(t)\right\| \leq M\left\|\varphi-\varphi^{*}\right\|_{1} \hat{e}_{\ominus_{\nu} \lambda}(t, 0),
$$

where $\left\|\varphi-\varphi^{*}\right\|_{1}=\sup _{t \in(-\infty, 0]_{\mathbb{T}}}\left\|\varphi(t)-\varphi^{*}(t)\right\|$.

\section{$\S 3$ Existence and uniqueness of almost-periodic solutions}

In this section, we establish sufficient conditions on the existence and uniqueness of almostperiodic solutions of $(1.2)$. For $k=1,2, \ldots, m, i=1,2, \ldots, n_{k}$, we denote:

$$
\begin{gathered}
\overline{a_{k i}}=\sup _{t \in[0,+\infty)_{\mathbb{T}}}\left|a_{k i}(t)\right|, \underline{a_{k i}}=\inf _{t \in[0,+\infty)_{\mathbb{T}}}\left|a_{k i}(t)\right|, \\
\overline{w_{p j}^{k i}}=\sup _{t \in[0,+\infty)_{\mathbb{T}}}\left|w_{p j}^{k i}(t)\right|, \overline{I_{k i}}=\sup _{t \in[0,+\infty)_{\mathbb{T}}}\left|I_{k i}(t)\right|, \underline{a}=\min _{\substack{k=1,2, \ldots, m \\
i=1,2, \ldots, n_{k}}} \underline{a_{k i}} .
\end{gathered}
$$

Throughout this paper, for any $k=1,2, \ldots, m, i=1,2, \ldots, n_{k}, p=1,2, \ldots, m, p \neq k, j=$ $1,2, \ldots, n_{p}$, we make the following assumptions:

$\left(H_{1}\right)$ the signal decay rate $a_{k i}(t)$ is a continuous almost periodic function on $[0,+\infty)_{\mathbb{T}}$, $\underline{a_{k i}}>0, a_{k i}(t) \in R_{\nu}^{+}$, the connection weight $w_{p j}^{k i}(t)$ and the network's external input $I_{k i}(t)$ are bounded continuous almost periodic functions on $[0,+\infty)_{\mathbb{T}}$;

$\left(H_{2}\right)$ the delay kernel function $g_{p j}^{k i}(s):[0,+\infty)_{\mathbb{T}} \rightarrow[0,+\infty)$ is piecewise continuous and satisfies $\int_{0}^{+\infty} g_{p j}^{k i}(s) \nabla s=1, \int_{0}^{+\infty} \hat{e}_{\underline{a}}(s, 0) g_{p j}^{k i}(s) \nabla s<+\infty$;

$\left(H_{3}\right)$ there exists a positive constant $L_{k i}$ such that $\left|f_{k i}(u)-f_{k i}(v)\right| \leq L_{k i}|u-v|$ for each $u, v \in \mathbb{R}$.

Define matrices

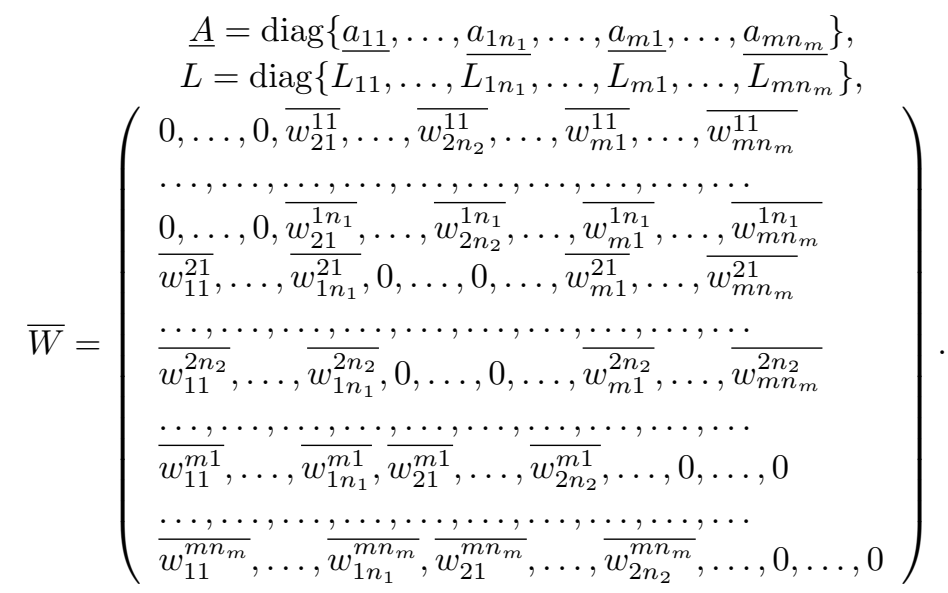

Now, we are in a position to state our first theorem.

Theorem 3.1 Assume $\left(H_{1}\right)-\left(H_{3}\right)$ hold, and $\widetilde{\rho}\left(\underline{A}^{-1} \bar{W} L\right)<1$. Then $(1.2)$ has a unique almostperiodic solution.

Proof. For any given $\varphi(t)=\operatorname{col}\left\{\varphi_{k i}(t)\right\} \in S$, we consider the following almost-periodic 
system:

$$
x_{k i}^{\nabla}(t)=-a_{k i}(t) x_{k i}(t)+\sum_{p=1, p \neq k}^{m} \sum_{j=1}^{n_{p}} w_{p j}^{k i}(t) f_{p j}\left(\int_{0}^{+\infty} g_{p j}^{k i}(s) \varphi_{p j}(t-s) \nabla s\right)+I_{k i}(t) .
$$

From $\left(H_{1}\right)$ and Lemma 2.5 , it follows that the linear system

$$
x_{k i}^{\nabla}(t)=-a_{k i}(t) x_{k i}(t), \quad k=1,2, \ldots, m, i=1,2, \ldots, n_{k}
$$

admits an exponential dichotomy. Thus, by Lemma 2.4, we see that (3.1) has a unique almostperiodic solution, which can be expressed as

$$
\begin{array}{r}
U_{\varphi}(t)=\operatorname{col}\left\{\int _ { - \infty } ^ { t } \hat { e } _ { - a _ { k i } } ( t , \rho ( u ) ) \left(\sum_{p=1, p \neq k}^{m} \sum_{j=1}^{n_{p}} w_{p j}^{k i}(u) f_{p j}\left(\int_{0}^{+\infty} g_{p j}^{k i}(s) \varphi_{p j}(u-s) \nabla s\right)\right.\right. \\
\left.\left.+I_{k i}(u)\right) \nabla u\right\} .
\end{array}
$$

Define an operator $T: S \rightarrow S$ by:

$$
T(\varphi(t))=U_{\varphi}(t), \quad \forall \varphi(t) \in S .
$$

We will show that $T$ is a contraction.

For any $\xi(t), \zeta(t) \in S$, we have

$$
\begin{gathered}
|T(\xi(t))-T(\zeta(t))|=\operatorname{col}\left\{\mid \int_{-\infty}^{t} \hat{e}_{-a_{k i}}(t, \rho(u)) \sum_{p=1, p \neq k}^{m} \sum_{j=1}^{n_{p}} w_{p j}^{k i}(u)\right. \\
\left.\left[f_{p j}\left(\int_{0}^{+\infty} g_{p j}^{k i}(s) \xi_{p j}(u-s) \nabla s\right)-f_{p j}\left(\int_{0}^{+\infty} g_{p j}^{k i}(s) \zeta_{p j}(u-s) \nabla s\right)\right] \nabla u \mid\right\} \\
\leq \operatorname{col}\left\{\int_{-\infty}^{t} \hat{e}_{-\underline{a_{k i}}}(t, \rho(u)) \sum_{p=1, p \neq k}^{m} \sum_{j=1}^{n_{p}} \overline{w_{p j}^{k i}} \mid f_{p j}\left(\int_{0}^{+\infty} g_{p j}^{k i}(s) \xi_{p j}(u-s) \nabla s\right)\right. \\
\left.-f_{p j}\left(\int_{0}^{+\infty} g_{p j}^{k i}(s) \zeta_{p j}(u-s) \nabla s\right) \mid \nabla u\right\} \\
\leq \operatorname{col}\left\{\int_{-\infty}^{t} \hat{e}_{-a_{k i}}(t, \rho(u)) \sum_{p=1, p \neq k}^{m} \sum_{j=1}^{n_{p}} \overline{w_{p j}^{k i}} L_{p j} \int_{0}^{+\infty} g_{p j}^{k i}(s) \mid \xi_{p j}(u-s)\right. \\
\left.-\zeta_{p j}(u-s) \mid \nabla s \nabla u\right\} \\
\leq \operatorname{col}\left\{\int_{-\infty}^{t} \hat{e}_{-\underline{a_{k i}}}(t, \rho(u)) \sum_{p=1, p \neq k}^{m} \sum_{j=1}^{n_{p}} \overline{w_{p j}^{k i}} L_{p j} \sup _{s \leq t}\left|\xi_{p j}(s)-\zeta_{p j}(s)\right| \nabla u\right\} \\
\leq \operatorname{col}\left\{\frac{1}{\underline{a_{k i}}} \sum_{p=1, p \neq k}^{m} \sum_{j=1}^{n_{p}} \overline{w_{p j}^{k i}} L_{p j} \sup _{s \leq t}\left|\xi_{p j}(s)-\zeta_{p j}(s)\right|\right\} \\
=\underline{A^{-1}} \bar{W} L \operatorname{col}\left\{\sup _{s \leq t}\left|\xi_{k i}(s)-\zeta_{k i}(s)\right|\right\}
\end{gathered}
$$

It follows that

$$
\begin{aligned}
\sup _{t \in[0,+\infty)_{\mathbb{T}}}|T(\xi(t))-T(\zeta(t))| & \leq \underline{A}^{-1} \bar{W} L \operatorname{col}\left\{\sup _{t \in[0,+\infty)_{\mathbb{T}}}\left|\xi_{k i}(t)-\zeta_{k i}(t)\right|\right\} \\
& =\underline{A}^{-1} \bar{W} L \sup _{t \in[0,+\infty)_{\mathbb{T}}}|\xi(t)-\zeta(t)| .
\end{aligned}
$$


By using (3.2) repeatedly, we obtain that

where $k$ is an arbitrary positive integer.

$$
\begin{aligned}
\sup _{t \in[0,+\infty)_{\mathbb{T}}}\left|T^{k}(\xi(t))-T^{k}(\zeta(t))\right| & =\sup _{t \in[0,+\infty)_{\mathbb{T}}}\left|T\left(T^{k-1}(\xi(t))\right)-T\left(T^{k-1}(\zeta(t))\right)\right| \\
& \leq \underline{A}^{-1} \bar{W} L \sup _{t \in[0,+\infty)_{\mathbb{T}}}\left|T^{k-1}(\xi(t))-T^{k-1}(\zeta(t))\right| \\
& \leq\left(\underline{A}^{-1} \bar{W} L\right)^{2} \sup _{t \in[0,+\infty)_{\mathbb{T}}}\left|T^{k-2}(\xi(t))-T^{k-2}(\zeta(t))\right| \\
& \leq \ldots \leq\left(\underline{A}^{-1} \bar{W} L\right)^{k} \sup _{t \in[0,+\infty)_{\mathbb{T}}}|\xi(t)-\zeta(t)|,
\end{aligned}
$$

Because $\widetilde{\rho}\left(\underline{A}^{-1} \bar{W} L\right)<1$, we can obtain that $\lim _{k \rightarrow \infty}\left(\underline{A}^{-1} \bar{W} L\right)^{k}=0_{N \times N}$. Therefore, for any $0<\varepsilon<1$, there exists a positive integer $K$ such that every element of the matrix $\left(\underline{A}^{-1} \bar{W} L\right)^{K}$ is smaller than $\frac{\varepsilon}{N}$. If we set $\left(\underline{A}^{-1} \bar{W} L\right)^{K}=\left(b_{p j}^{k i}\right)_{N \times N}$, then $\sum_{k=1}^{m} \sum_{i=1}^{n_{k}} b_{p j}^{k i}<\sum_{k=1}^{m} \sum_{i=1}^{n_{k}} \frac{\varepsilon}{N}=\varepsilon$.

From (3.3), we have

$$
\begin{aligned}
\sup _{t \in[0,+\infty)_{\mathbb{T}}}\left|T^{K}(\xi(t))-T^{K}(\zeta(t))\right| & \leq \operatorname{col}\left\{\sum_{k=1}^{m} \sum_{i=1}^{n_{k}} b_{p j}^{k i} \sup _{t \in[0,+\infty)_{\mathbb{T}}}\left|\xi_{k i}(t)-\zeta_{k i}(t)\right|\right\} \\
& \leq \sup _{t \in[0,+\infty)_{\mathbb{T}}} \max _{\substack{k=1,2, \ldots, m \\
i=1,2, \ldots, n_{k}}}\left|\xi_{k i}(t)-\zeta_{k i}(t)\right| \operatorname{col}\left\{\sum_{k=1}^{m} \sum_{i=1}^{n_{k}} b_{p j}^{k i}\right\}
\end{aligned}
$$

which implies that $\left\|T^{K}(\xi)-T^{K}(\zeta)\right\| \leq \varepsilon\|\xi-\zeta\|$.

Because $0<\varepsilon<1, T^{K}$ is a contraction mapping from $S$ to $S$. According to Banach fixed point theorem, there exists a unique fixed point $\varphi^{*} \in S$ such that $T^{K}\left(\varphi^{*}\right)=\varphi^{*}$. It follows that $T^{K}\left(T\left(\varphi^{*}\right)\right)=T\left(T^{K}\left(\varphi^{*}\right)\right)=T\left(\varphi^{*}\right)$, which implies that $T\left(\varphi^{*}\right) \in S$ is also a fixed point of map $T^{K}$. By the uniqueness of fixed points, we have that $T\left(\varphi^{*}\right)=\varphi^{*}$ and then $\varphi^{*}$ satisfies (1.2). Hence $\varphi^{*}$ is the unique almost periodic solution of (1.2) in $S$.

\section{$\S 4$ Exponential stability of almost-periodic solutions}

In this section, we derive sufficient conditions for the exponential stability of almost-periodic solutions of (1.2).

Theorem 4.1 Assume $\left(H_{1}\right)-\left(H_{3}\right)$ hold and $\widetilde{\rho}\left(\underline{A}^{-1} \bar{W} L\right)<1$. Moreover, assume that

$\left(H_{4}\right)$ there exists a positive constant $\alpha>0, \alpha \in \mathcal{R}_{\nu}^{+}$such that for $t \in(0, \infty)_{\mathbb{T}}, k=$ $1,2, \ldots, m, i=1,2, \ldots, n_{k}$,

$$
\begin{aligned}
& \alpha+\left(2{\overline{a_{k i}}}^{2} \nu(t)-\underline{a_{k i}}\right)(1-\nu(t) \alpha) \\
& \quad+\left(1+2 \overline{a_{k i}} \nu(t)\right)(1-\nu(t) \alpha) \sum_{p=1, p \neq k}^{m} \sum_{j=1}^{n_{p}} \overline{w_{p j}^{k i}} L_{p j} \int_{0}^{+\infty} \hat{e}_{\alpha}(t, t-s) g_{p j}^{k i}(s) \nabla s<0 .
\end{aligned}
$$

Then (1.2) has an almost-periodic solution which is globally exponentially stable.

Proof. Theorem 3.1 tells us that (1.2) has an almost-periodic solution. Let $x^{*}=\operatorname{col}\left\{x_{k i}^{*}(t)\right\}$ be this solution with initial value $\varphi^{*}(s)=\operatorname{col}\left\{\varphi_{k i}^{*}(s)\right\}, s \in(-\infty, 0]_{\mathbb{T}}$. We show that $x^{*}$ is globally exponentially stable.

Let $x(t)=\operatorname{col}\left\{x_{k i}(t)\right\}$ be an arbitrary solution of (1.2) with initial value $\varphi(s)=\operatorname{col}\left\{\varphi_{k i}(s)\right\}$, 
$s \in(-\infty, 0]_{\mathbb{T}}$. Define

for $k=1,2, \ldots, m, i=1,2, \ldots, n_{k}$. It follows that

$$
u_{k i}(t)=x_{k i}(t)-x_{k i}^{*}(t)
$$

$$
\begin{aligned}
u_{k i}^{\nabla}(t)= & -a_{k i}(t) u_{k i}(t)+\sum_{p=1, p \neq k}^{m} \sum_{j=1}^{n_{p}} w_{p j}^{k i}(t)\left(f_{p j}\left(\int_{0}^{+\infty} g_{p j}^{k i}(s) x_{p j}(t-s) \nabla s\right)\right. \\
& \left.-f_{p j}\left(\int_{0}^{+\infty} g_{p j}^{k i}(s) x_{p j}^{*}(t-s) \nabla s\right)\right) \\
= & -a_{k i}(t) u_{k i}(t)+\sum_{p=1, p \neq k}^{m} \sum_{j=1}^{n_{p}} w_{p j}^{k i}(t)\left[f _ { p j } \left(\int_{0}^{+\infty} g_{p j}^{k i}(s) x_{p j}(t-s) \nabla s\right.\right. \\
& \left.-\int_{0}^{+\infty} g_{p j}^{k i}(s) x_{p j}^{*}(t-s) \nabla s+\int_{0}^{+\infty} g_{p j}^{k i}(s) x_{p j}^{*}(t-s) \nabla s\right) \\
& \left.-f_{p j}\left(\int_{0}^{+\infty} g_{p j}^{k i}(s) x_{p j}^{*}(t-s) \nabla s\right)\right] \\
= & -a_{k i}(t) u_{k i}(t)+\sum_{p=1, p \neq k}^{m} \sum_{j=1}^{n_{p}} w_{p j}^{k i}(t)\left[f _ { p j } \left(\int_{0}^{+\infty} g_{p j}^{k i}(s) u_{p j}(t-s) \nabla s\right.\right. \\
& \left.\left.+\int_{0}^{+\infty} g_{p j}^{k i}(s) x_{p j}^{*}(t-s) \nabla s\right)-f_{p j}\left(\int_{0}^{+\infty} g_{p j}^{k i}(s) x_{p j}^{*}(t-s) \nabla s\right)\right] .
\end{aligned}
$$

We denote

$$
\begin{aligned}
& F_{p j}\left(\int_{0}^{+\infty} g_{p j}^{k i}(s) u_{p j}(t-s) \nabla s\right) \\
& =f_{p j}\left(\int_{0}^{+\infty} g_{p j}^{k i}(s) u_{p j}(t-s) \nabla s+\int_{0}^{+\infty} g_{p j}^{k i}(s) x_{p j}^{*}(t-s) \nabla s\right) \\
& \quad-f_{p j}\left(\int_{0}^{+\infty} g_{p j}^{k i}(s) x_{p j}^{*}(t-s) \nabla s\right) .
\end{aligned}
$$

Then

$$
u_{k i}^{\nabla}(t)=-a_{k i}(t) u_{k i}(t)+\sum_{p=1, p \neq k}^{m} \sum_{j=1}^{n_{p}} w_{p j}^{k i}(t) F_{p j}\left(\int_{0}^{+\infty} g_{p j}^{k i}(s) u_{p j}(t-s) \nabla s\right) .
$$

From $\left(H_{3}\right)$, we have

for any $u_{p j}(t): \mathbb{T} \rightarrow \mathbb{R}$ and $F_{p j}(0)=0$.

$$
\left|F_{p j}\left(\int_{0}^{+\infty} g_{p j}^{k i}(s) u_{p j}(t-s) \nabla s\right)\right| \leq L_{p j}\left|\int_{0}^{+\infty} g_{p j}^{k i}(s) u_{p j}(t-s) \nabla s\right|,
$$

The initial conditions associated with (4.1) are of the form

$$
u_{k i}(s)=\psi_{k i}(s)=\varphi_{k i}(s)-\varphi_{k i}^{*}(s),
$$

where $k=1,2, \ldots, m, i=1,2, \ldots, n_{k}, s \in(-\infty, 0]_{\mathbb{T}}$. 
By calculating the Dini derivative of $u_{k i}$ along the solutions of system (4.1), we have

$$
\begin{aligned}
D^{-} & \left(\left|u_{k i}(t)\right|^{\nabla}\right) \\
\leq & \operatorname{sign}\left(u_{k i}(\rho(t))\right) u_{k i}^{\nabla}(t) \\
\leq & -a_{k i}(t) u_{k i}(t) \operatorname{sign}\left(u_{k i}(\rho(t))\right)+\sum_{p=1, p \neq k}^{m} \sum_{j=1}^{n_{p}} \overline{w_{p j}^{k i}} L_{p j} \int_{0}^{+\infty} g_{p j}^{k i}(s)\left|u_{p j}(t-s)\right| \nabla s \\
= & -a_{k i}(t) \operatorname{sign}\left(u_{k i}(\rho(t))\right)\left(u_{k i}(\rho(t))+\nu(t) u_{k i}^{\nabla}(t)\right) \\
& +\sum_{p=1, p \neq k}^{m} \sum_{j=1}^{n_{p}} \overline{w_{p j}^{k i}} L_{p j} \int_{0}^{+\infty} g_{p j}^{k i}(s)\left|u_{p j}(t-s)\right| \nabla s \\
\leq & -\underline{a_{k i}}\left|u_{k i}(\rho(t))\right|+\overline{a_{k i}} \nu(t)\left|u_{k i}^{\nabla}(t)\right|+\sum_{p=1, p \neq k}^{m} \sum_{j=1}^{n_{p}} \overline{w_{p j}^{k i}} L_{p j} \int_{0}^{+\infty} g_{p j}^{k i}(s)\left|u_{p j}(t-s)\right| \nabla s \\
= & -\underline{a_{k i}}\left|u_{k i}(t)-\nu(t) u_{k i}^{\nabla}(t)\right|+\overline{a_{k i}} \nu(t)\left|u_{k i}^{\nabla}(t)\right| \\
& +\sum_{p=1, p \neq k}^{m} \sum_{j=1}^{n_{p}} \overline{w_{p j}^{k i}} L_{p j} \int_{0}^{+\infty} g_{p j}^{k i}(s)\left|u_{p j}(t-s)\right| \nabla s \\
\leq & -\underline{a_{k i}}\left|u_{k i}(t)\right|+2 \overline{a_{k i}} \nu(t)\left|u_{k i}^{\nabla}(t)\right|+\sum_{p=1, p \neq k}^{m} \sum_{j=1}^{n_{p}} \overline{w_{p j}^{k i}} L_{p j} \int_{0}^{+\infty} g_{p j}^{k i}(s)\left|u_{p j}(t-s)\right| \nabla s \\
\leq & \left(2 \overline{a_{k i}}{ }^{2} \nu(t)-\underline{a_{k i}}\right)\left|u_{k i}(t)\right|+\left(1+2 \overline{a_{k i}} \nu(t)\right) \sum_{p=1, p \neq k}^{m} \sum_{j=1}^{n_{p}} \overline{w_{p j}^{k i}} L_{p j} \int_{0}^{+\infty} g_{p j}^{k i}(s)\left|u_{p j}(t-s)\right| \nabla s .
\end{aligned}
$$

Set $y_{k i}(t)=\hat{e}_{\alpha}(t, 0)\left|u_{k i}(t)\right|$. By calculating the Dini derivative of $y_{k i}$ along the solutions of system (4.1), we have

$$
\begin{aligned}
& D^{-} y_{k i}^{\nabla}(t) \\
&= \alpha \hat{e}_{\alpha}(t, 0)\left|u_{k i}(t)\right|+\hat{e}_{\alpha}(\rho(t), 0) D^{-}\left(\left|u_{k i}(t)\right|^{\nabla}\right) \\
& \leq\left(\alpha \hat{e}_{\alpha}(t, 0)+\left(2 \bar{a}_{k i}^{2} \nu(t)-\underline{a_{k i}}\right)(1-\nu(t) \alpha) \hat{e}_{\alpha}(t, 0)\right)\left|u_{k i}(t)\right| \\
&+\left(1+2 \overline{a_{k i}} \nu(t)\right)(1-\nu(t) \alpha) \hat{e}_{\alpha}(t, 0) \sum_{p=1, p \neq k}^{m} \sum_{j=1}^{n_{p}} \overline{w_{p j}^{k i}} L_{p j} \int_{0}^{+\infty} g_{p j}^{k i}(s)\left|u_{p j}(t-s)\right| \nabla s \\
& \leq\left(\alpha+\left(2 \bar{a}_{k i}^{2} \nu(t)-\underline{a_{k i}}\right)(1-\nu(t) \alpha)\right)\left|y_{k i}(t)\right| \\
&+\left(1+2 \overline{a_{k i}} \nu(t)\right)(1-\nu(t) \alpha) \sum_{p=1, p \neq k}^{m} \sum_{j=1}^{n_{p}} \overline{w_{p j}^{k i}} L_{p j} \int_{0}^{+\infty} \hat{e}_{\alpha}(t, t-s) g_{p j}^{k i}(s) \nabla s \sup _{-\infty<s \leq t} y_{p j}(s) .
\end{aligned}
$$

Set $l_{0}=(1+\delta)\|\psi\|_{1}$, where $\delta$ is a positive constant.

When $s \in(-\infty, 0]_{\mathbb{T}}$, we have

$$
y_{k i}(s)=\hat{e}_{\alpha}(s, 0)\left|\psi_{k i}(s)\right|=\frac{1}{\hat{e}_{\alpha}(0, s)}\left|\psi_{k i}(s)\right| \leq\|\psi\|_{1}<l_{0},
$$

where $k=1,2, \ldots, m, i=1,2, \ldots, n_{k}$.

Next, we claim that

$$
y_{k i}(t)<l_{0}, \quad t \in(0,+\infty)_{\mathbb{T}}, k=1,2, \ldots, m, i=1,2, \ldots, n_{k} .
$$

By contradiction, if (4.3) is not true, there exist $k, i, t_{1}>0$ such that $y_{k i}\left(t_{1}\right) \geq l_{0}$, $D^{-} y_{k i}^{\nabla}\left(t_{1}\right) \geq 0$, and $y_{k i}(t)<l_{0}$ for $t \in\left(-\infty, t_{1}\right)_{\mathbb{T}}$. 
From $\left(H_{4}\right)$ and $(4.2)$, we obtain

$$
\begin{aligned}
D^{-} y_{k i}^{\nabla}\left(t_{1}\right) \leq & {\left[\alpha+\left(2{\overline{a_{k i}}}^{2} \nu(t)-\underline{a_{k i}}\right)(1-\nu(t) \alpha)\right.} \\
& \left.+\left(1+2 \overline{a_{k i}} \nu(t)\right)(1-\nu(t) \alpha) \sum_{p=1, p \neq k}^{m} \sum_{j=1}^{n_{p}} \overline{w_{p j}^{k i}} L_{p j} \int_{0}^{+\infty} \hat{e}_{\alpha}(t, t-s) g_{p j}^{k i}(s) \nabla s\right] l_{0} \\
< & 0,
\end{aligned}
$$

which conflicts with $D^{-} y_{k i}^{\nabla}\left(t_{1}\right) \geq 0$. Hence, (4.3) holds.

It follows that where $M=1+\delta>1$.

$$
\left|u_{k i}(t)\right|<l_{0} \hat{e}_{\ominus_{\nu} \alpha}(t, 0)=(1+\delta)\|\psi\|_{1} \hat{e}_{\ominus_{\nu} \alpha}(t, 0)=M\|\psi\|_{1} \hat{e}_{\ominus_{\nu} \alpha}(t, 0),
$$

Therefore, the almost-periodic solution $x^{*}$ of $(1.2)$ is globally exponentially stable. This completes the proof.

\section{$\S 5$ Applications}

In this section, we present an example to illustrate the feasibility and effectiveness of our results obtained in the previous sections for $\mathbb{T}=\mathbb{Z}$.

Consider the following MAM neutral network with $m=3, n_{1}=1, n_{2}=1, n_{3}=2$,

$$
\left\{\begin{aligned}
x_{11}^{\nabla}(t)= & -a_{11}(t) x_{11}(t)+w_{21}^{11}(t) f_{21}\left(\int_{0}^{+\infty} g_{21}^{11}(s) x_{21}(t-s) \nabla s\right) \\
& +w_{31}^{11}(t) f_{31}\left(\int_{0}^{+\infty} g_{31}^{11}(s) x_{31}(t-s) \nabla s\right) \\
& +w_{32}^{11}(t) f_{32}\left(\int_{0}^{+\infty} g_{32}^{11}(s) x_{32}(t-s) \nabla s\right)+I_{11}(t) \\
x_{21}^{\nabla}(t)= & -a_{21}(t) x_{21}(t)+w_{11}^{21}(t) f_{11}\left(\int_{0}^{+\infty} g_{11}^{21}(s) x_{11}(t-s) \nabla s\right) \\
& +w_{31}^{21}(t) f_{31}\left(\int_{0}^{+\infty} g_{31}^{21}(s) x_{31}(t-s) \nabla s\right) \\
& +w_{32}^{21}(t) f_{32}\left(\int_{0}^{+\infty} g_{32}^{21}(s) x_{32}(t-s) \nabla s\right)+I_{21}(t) \\
x_{31}^{\nabla}(t)= & -a_{31}(t) x_{31}(t)+w_{11}^{31}(t) f_{11}\left(\int_{0}^{+\infty} g_{11}^{31}(s) x_{11}(t-s) \nabla s\right) \\
& +w_{21}^{31}(t) f_{21}\left(\int_{0}^{+\infty} g_{21}^{31}(s) x_{21}(t-s) \nabla s\right)+I_{31}(t) \\
x_{32}^{\nabla}(t)= & -a_{32}(t) x_{32}(t)+w_{11}^{32}(t) f_{11}\left(\int_{0}^{+\infty} g_{11}^{32}(s) x_{11}(t-s) \nabla s\right) \\
& +w_{21}^{32}(t) f_{21}\left(\int_{0}^{+\infty} g_{21}^{32}(s) x_{21}(t-s) \nabla s\right)+I_{32}(t),
\end{aligned}\right.
$$

where the neuronal signal decay rates $a_{k i}(t)=\frac{1}{4}, k=1,2, \ldots, m, i=1,2, \ldots, n_{k}$, the external inputs

$$
I_{11}(t)=2+\sin t, I_{21}(t)=2+2 \cos t, I_{31}(t)=2+\sin (2 t), I_{32}(t)=2+\cos (2 t) .
$$

The connection weights are set to be constants as in the following

$$
W=\left(\begin{array}{lrrr}
0 & w_{21}^{11} & w_{31}^{11} & w_{32}^{11} \\
w_{11}^{21} & 0 & w_{31}^{21} & w_{32}^{21} \\
w_{11}^{31} & w_{21}^{31} & 0 & 0 \\
w_{11}^{32} & w_{21}^{32} & 0 & 0
\end{array}\right)
$$




$$
=\left(\begin{array}{lrrc}
0 & 0.02 & 0.01 & 0.02 \\
0.02 & 0 & 0.03 & 0.01 \\
0.01 & 0.01 & 0 & 0 \\
0.02 & 0.03 & 0 & 0
\end{array}\right) .
$$

The neuronal activation functions $f_{p j}(x)=\frac{1}{20} \sin \left(\frac{3}{4} x\right)$, and the delay kernel functions $g_{p j}^{k i}(t)=$ $\frac{1}{2^{t}}$.

We can take the initial value $\theta_{11}(s)=0.3, \theta_{21}(s)=0.25, \theta_{31}(s)=0.1, \theta_{32}(s)=0.2$, for $s \in(-\infty, 0]_{\mathbb{T}}$.

It is not difficult to obtain that $a_{k i}(t)$ are continuous almost periodic functions on $[0,+\infty)_{\mathbb{T}}$, $w_{p j}^{k i}(t)$ and $I_{k i}(t)$ are bounded continuous almost periodic functions on $[0,+\infty)_{\mathbb{T}}$, and $\underline{a_{k i}}>$ $0, a_{k i}(t) \in R_{\nu}^{+}, g_{p j}^{k i}(s):[0,+\infty)_{\mathbb{T}} \rightarrow[0,+\infty)$ are piecewise continuous, and satisfy $\int_{0}^{+\infty} g_{p j}^{k i}(s) \nabla s$ $=1$, and $\int_{0}^{+\infty} \hat{e}_{\underline{a}}(s, 0) g_{p j}^{k i}(s) \nabla s=2<+\infty, L_{k i}=\frac{1}{20}$. Therefore, the matrix $\underline{A}=\operatorname{diag}\{0.25,0.25$, $0.25,0.25\}, L=\operatorname{diag}\{0.05,0.05,0.05,0.05\}, \bar{W}=W$,

$$
\underline{A}^{-1} \bar{W} L=\left(\begin{array}{cccc}
0 & 0.004 & 0.002 & 0.004 \\
0.004 & 0 & 0.006 & 0.002 \\
0.002 & 0.002 & 0 & 0 \\
0.004 & 0.006 & 0 & 0
\end{array}\right),
$$

$\widetilde{\rho}\left(\underline{A}^{-1} \bar{W} L\right)=0.0091<1$.

Let $\alpha=0.1$, we have

$$
\begin{aligned}
\alpha+ & \left(2{\overline{a_{11}}}^{2} \nu(t)-\underline{a_{11}}\right)(1-\nu(t) \alpha) \\
& +\left(1+2 \overline{a_{11}} \nu(t)\right)(1-\nu(t) \alpha) \sum_{p=1, p \neq k}^{3} \sum_{j=1}^{n_{p}} \overline{w_{p j}^{11}} L_{p j} \int_{0}^{+\infty} \hat{e}_{\alpha}(t, t-s) g_{p j}^{11}(s) \nabla s \\
= & \alpha+\left(2 \bar{a}_{11}^{2} \nu(t)-\underline{a_{11}}\right)(1-\nu(t) \alpha) \\
& +\left(1+2 \overline{a_{11}} \nu(t)\right)(1-\nu(t) \alpha)\left(\overline{w_{21}^{11}} L_{21} \int_{0}^{+\infty} \hat{e}_{\alpha}(t, t-s) g_{21}^{11}(s) \nabla s\right. \\
& \left.+\overline{w_{31}^{11}} L_{31} \int_{0}^{+\infty} \hat{e}_{\alpha}(t, t-s) g_{31}^{11}(s) \nabla s+\overline{w_{32}^{11}} L_{32} \int_{0}^{+\infty} \hat{e}_{\alpha}(t, t-s) g_{32}^{11}(s) \nabla s\right) \\
= & 0.1+\left(\frac{1}{8}-\frac{1}{4}\right)(1-0.1)+2\left(1+\frac{1}{2}\right)(1-0.1)\left(0.05 \cdot \frac{1}{20}\right) \\
= & -0.01<0 .
\end{aligned}
$$

Similarly, we can get

$$
\begin{aligned}
\alpha+ & \left(2{\overline{a_{21}}}^{2} \nu(t)-\underline{a_{21}}\right)(1-\nu(t) \alpha) \\
& +\left(1+2 \overline{a_{21}} \nu(t)\right)(1-\nu(t) \alpha) \sum_{p=1, p \neq 2}^{3} \sum_{j=1}^{n_{p}} \overline{w_{p j}^{21}} L_{p j} \int_{0}^{+\infty} \hat{e}_{\alpha}(t, t-s) g_{p j}^{21}(s) \nabla s \\
= & -0.01<0, \\
\alpha+ & \left(2{\overline{a_{31}}}^{2} \nu(t)-\underline{a_{31}}\right)(1-\nu(t) \alpha) \\
& +\left(1+2 \overline{a_{31}} \nu(t)\right)(1-\nu(t) \alpha) \sum_{p=1, p \neq 3}^{3} \sum_{j=1}^{n_{p}} \overline{w_{p j}^{31}} L_{p j} \int_{0}^{+\infty} \hat{e}_{\alpha}(t, t-s) g_{p j}^{31}(s) \nabla s \\
= & -0.004<0,
\end{aligned}
$$




$$
\begin{aligned}
\alpha+ & \left(2{\overline{a_{32}}}^{2} \nu(t)-\underline{a_{32}}\right)(1-\nu(t) \alpha) \\
& +\left(1+2 \overline{a_{32}} \nu(t)\right)(1-\nu(t) \alpha) \sum_{p=1, p \neq 3}^{3} \sum_{j=1}^{n_{p}} \overline{w_{p j}^{32}} L_{p j} \int_{0}^{+\infty} \hat{e}_{\alpha}(t, t-s) g_{p j}^{32}(s) \nabla s \\
= & -0.01<0 .
\end{aligned}
$$

Then $\left(H_{1}\right)-\left(H_{4}\right)$ are all satisfied.

By Theorem 3.1, system (5.1) has exactly one almost-periodic solution. Moreover, by Theorem 4.1 this solution is globally exponentially stable.

\section{$\S 6$ Conclusions}

In this paper, we expand a MAM neural networks with distributed delays to time scales. We establish some sufficient conditions on the existence, uniqueness and the global exponential stability of almost-periodic solutions. An example is presented to illustrate the feasibility and effectiveness of the obtained results for $\mathbb{T}=\mathbb{Z}$. We use Banach fixed point theorem, inequality method and reduction to absurdity in this paper. In recent years, only little attention has been paid towards almost-periodic solutions to MAM neural networks on time scales, we will study more deeply about it in the future.

\section{Acknowledgement}

Our deepest gratitude goes to the anonymous reviewers for their careful work and thoughtful suggestions that have helped improve this paper substantially.

Open Access This article is licensed under a Creative Commons Attribution 4.0 International License, which permits use, sharing, adaptation, distribution and reproduction in any medium or format, as long as you give appropriate credit to the original author(s) and the source, provide a link to the Creative Commons licence, and indicate if changes were made. The images or other third party material in this article are included in the articles Creative Commons licence, unless indicated otherwise in a credit line to the material. If material is not included in the articles Creative Commons licence and your intended use is not permitted by statutory regulation or exceeds the permitted use, you will need to obtain permission directly from the copyright holder. To view a copy of this licence, visit http://creativecomm ons.org/licenses/by/4.0/.

\section{References}

[1] D Anderson, J Bullock, L Erbe, A Peterson, H N Tran. Nabla dynamic equations on time scales, Panamer Mathematics Journal, 2003, 13(1): 1-47.

[2] M Bohner, A Peterson. Dynamic equations on time Scales: An introduction with applications, Birkhäuser Boston, Boston, 2001. 
[3] M Bohner, A Peterson. Advances in dynamic equations on time scales, Birkhäuser Boston, Boston, 2003.

[4] J D Cao, J Wang. Global asymptotic and robust stability of recurrent neural networks with time delays, IEEE Trans Circuits Syst I, Reg Papers, 2005, 52(2): 417-426.

[5] J D Cao, J Wang. Global exponential stability and periodicity of recurrent neural networks with time delays, IEEE Trans Circuits Syst I, Reg Papers, 2005, 52(2): 920-931.

[6] J D Cao, J L Liang. Boundedness and stability for Cohen-Grossberg neural network with timevarying delays, J Math Anal \& App, 2004, 296(2): 665-685.

[7] A M Fink. Almost periodic differential equations, Lecture Notes in Mathematics, Springer-Verlag, Berlin-New York, 1974

[8] J Gao, Q R Wang, L W Zhang. Existence and stability of almost-periodic solutions for cellular neural networks with time-varying delays in leakage terms on time scales, Appl Math Comput, 2014, 237: 639-649.

[9] J Gao, Q R Wang, Y Lin. Existence and exponential stability of almost-periodic solutions for neutral BAM neural networks with time-varying delays in leakage terms on time scales, Math Methods Appl Sci, 2016, 39(6): 1361-1375.

[10] M Hagiwara. Multidirectional associative memory, Proc Int Joint Conf Neural Netw, 1990, 1: 3-6.

[11] M Hattori, M Hagiwara. Multimodule associative memory for many-to-many associations, Neurocomputing, 1998, 19(1-3): 99-119.

[12] Y K Li, B Li. Almost periodic time scales and almost periodic functions on time scales, J Appl Math, 2015, 2015: Article ID 730672, 8 pp.

[13] Y K Li, C Wang. Almost periodic functions on time scales and applications, Discrete Dyn Nat Soc, 2011, 2011: Article ID 727068, 20 pp.

[14] Y K Li, C Wang. Uniformly almost periodic functions and almost periodic solutions to dynamic equations on time scales, Abstr Appl Anal, 2011, 2011: Article ID 341520, 22 pp.

[15] M Wang, T J Zhou. Multistability in a multidirectional associative memory neural network with delays, J Appl Math, 2013, 2013: Article ID 592056, 10 pp.

[16] T J Zhou, M Wang, C Li. Almost periodic solution for multidirectional associative memory neural network with distributed delays, Math Comput Simulat, 2015, 107(C): 52-60.

[17] T J Zhou, M Wang, M Long. Existence and exponential stability of multiple periodic solutions for a multidirectional associative memory neural network, Neural Process Lett, 2012, 35(2): 187-202.

${ }^{1}$ School of Computer Sciences, Shenzhen Institute of Information Technology, Shenzhen 518172,

China.

Email: 306996140@qq.com

${ }^{2}$ School of Mathematics, Sun Yat-sen University, Guangzhou 510275, China.

Email: mcswqr@mail.sysu.edu.cn

${ }^{3}$ Department of Public Courses, Shenzhen Institute of Information Technology, Shenzhen 518172, China.

Email: 229617098@qq.com 